\title{
GROUND STATE ENERGY OF THE LOW DENSITY BOSE GAS
}

\author{
Elliott H. Lieb ${ }^{1}$ and Jakob Yngvason ${ }^{2}$ \\ 1. Department of Physics, Jadwin Hall, Princeton University, P. O. Box 708, Princeton, New Jersey 08544 \\ 2. Institut für Theoretische Physik, Universität Wien, Boltzmanngasse 5, A 1090 Vienna, Austria
}

(27 October, 1997,)

\begin{abstract}
Now that the properties of low temperature Bose gases at low density, $\rho$, can be examined experimentally it is appropriate to revisit some of the formulas deduced by many authors 4-5 decades ago. One of these is that the leading term in the energy/particle is $2 \pi \hbar^{2} \rho a / m$, where $a$ is the scattering length. Owing to the delicate and peculiar nature of bosonic correlations, four decades of research have failed to establish this plausible formula rigorously. The only known lower bound for the energy was found by Dyson in 1957, but it was 14 times too small. The correct bound is proved here.
\end{abstract}

PACS numbers: 05.30.Jp, 03.75.Fi, 67.40-w

With the renewed experimental interest in low density, low temperature Bose gases, some of the formulas posited four and five decades ago have been dusted off and reexamined. One of these is the leading term in the ground state energy. In the limit of small particle density, $\rho$,

$$
e_{0}(\rho) \approx \mu 4 \pi \rho a
$$

where $e_{0}(\rho)$ is the ground state energy (g.s.e.) per particle in the thermodynamic limit, $a$ is the scattering length (assumed positive) of the two-body potential $v$ for bosons of mass $m$, and $\mu \equiv \hbar^{2} / 2 m$.

Is (i1) correct? In particular, is it true for the hardsphere gas? While there have been many attempts at a rigorous proof of (1) in the past forty years, none has been found so far. Our aim here is to supply that proof for finite range, positive potentials. As remarked below, (11) cannot hold unrestrictedly; more than $a>0$ is needed.

An upper bound for $e_{0}(\rho)$ agreeing with (11) is not easy to derive, but it was achieved for hard spheres by a variational calculation [1], which can be extended to include general, positive potentials of finite range. What remained unknown was a good lower bound. The only one available is Dyson's [1], and that is about fourteen times smaller than (11). In this paper we shall provide a lower bound of the desired form, and thus prove (11). We can also give explicit error bounds for small enough values of the dimensionless parameter $Y \equiv 4 \pi \rho a^{3} / 3$ :

$$
e_{0}(\rho) \geq \mu 4 \pi \rho a\left(1-C Y^{1 / 17}\right)
$$

for some fixed $C$ (which is not evaluated explicitly because $C$ and the exponent $1 / 17$ are only of academic interest). The bound (2) holds for all non-negative, finite range, spherical, two-body potentials. A typical experimental value [2] is $Y \approx 10^{-5}$. Dyson's upper bound is $\mu 4 \pi \rho a\left(1+2 Y^{1 / 3}\right)\left(1-Y^{1 / 3}\right)^{-2}$.

We conjecture that (11) requires only a positive scattering length and the absence of any many-body, negative energy bound state. If there are such bound states then (11) is certainly wrong, but this obvious caveat does not seem to have been clearly emphasized before. There is a 'nice' potential with positive scattering length, no 2-body bound state, but with a 3-body bound state [3.

Our method also obviously applies to the positive temperature free energy (because Neumann boundary conditions give an upper bound to the solution to the heat (or Bloch) equation).

We also give some explicit bounds for finite systems, which might be useful for experiments with traps, but we concentrate here on the thermodynamic limit for simplicity. For traps with slowly varying confining potentials, $V_{\text {ext }}$, our method will prove that the leading term in the energy is given by the well known local density approximation [4], which minimizes the gaseous energy (11) plus the confining energy, with respect to $\rho(\mathbf{x})$, namely,

$$
\mathcal{E}(\rho) \equiv \int\left(V_{\text {ext }}(\mathbf{x}) \rho(\mathbf{x})+\mu 4 \pi a \rho(\mathbf{x})^{2}\right) d^{3} \mathbf{x}
$$

is minimized subject to $\int \rho=N=$ number of particles.

The fact that Dyson's lower bound was not improved for four decades, despite many attempts, attests to the fact that bosons are subtle quantum mechanical objects which can have peculiar correlations unknown to fermions. For example, there is the non-thermodynamic $N^{7 / 5}$ law for the charged Bose gas that was discovered by Dyson [5], confirmed only 20 years later [6], and which defies any simple physical interpretation.

The first understanding of (11) goes back to Bogoliubov [7], who also introduced the notion of 'pairing' in Helium (which resurfaced in the BCS theory for fermions). Later, there were several derivations of (11) (and higher order) [8], 9]. The method of the pseudopotential, which is an old idea of Fermi's, was closest to the Bogoliubov analysis. The 'exact' pseudopotential was constructed in [10], but it did not help to make this appealing idea more rigorous. Most of the derivations were in momentum space, the exception being [9], which works directly in physical space and which can handle both long and short range potentials. See 111 for a review. All these methods rely on special assumptions about the ground state (e.g., se- 
lecting special terms in a perturbation expansion, which likely diverges) and it is important to derive a fundamental result like (1) without extra assumptions.

In all of this earlier work one key fact was not understood, or at least not clearly stated in connection with the derivation of (11). It is that there are two different regimes, even at low density, with very different physics, even though the simple formula (11) seems to depend only on the scattering length. Recall that the (two-body) scattering length is defined, for a spherically symmetric potential, $v$, by

$$
-\mu u_{0}^{\prime \prime}(r)+\frac{1}{2} v(r) u_{0}(r)=0
$$

with $u_{0}(0)=0, u_{0}(r)>0$ (which is equivalent to the absence of negative energy bound states, and which is true for nonnegative $v$ ). As $r \rightarrow \infty, u(r) \approx r-a$. (Note the $v / 2$ and not $v$ in (3) because of the reduced mass.) Thus, $a$ depends on $m$ in a nontrivial way, and there are two extremes:

1. Potential Energy Dominated Region: The hard sphere $(v(r)=\infty$ for $r<a)$, is the extreme case here; the scattering length is independent of $m$, and the energy is mostly (entirely) kinetic. We see this from (11) because $-m \partial e_{0} / \partial m$ is the kinetic energy (Hellmann-Feynman theorem). In this regime the potential is so dominant that it forces the energy to be mostly kinetic. The g.s. wave function is highly correlated.

2. Kinetic Energy Dominated Region: The typical case is a very 'soft' potential. Then $a \approx\left(m / \hbar^{2}\right) \int_{0}^{\infty} v(r) r^{2} d r$, which implies, from (1), that $e_{0}$ hardly depends on $m$. Thus, the energy is almost all potential. The g.s. wave function is essentially the noninteracting one in this limit.

In other words, 'scattering length' is not a property of $v$ alone, and the low density gas, viewed from the perspective of the bosons, looks quite different in the two regimes. Nevertheless, as (11) says, the energy cannot distinguish the two cases. Whether Bose-Einstein condensation itself can notice the difference remains to be seen. Condensation will not be touched upon here, except to note that so far the only case with 2-body interactions in which B-E condensation has been rigorously established is hard core lattice bosons, but only at half filling [12].

Dyson [1] effectively converted region 1 into region 2. We shall make use of his important idea, which substitutes a very soft potential for the original one (even a hard core) at the price of sacrificing the kinetic energy,

We assume that the $N$ particles are in a $L \times L \times L$ cubic box, $\Omega$. The particle density is then $\rho=N L^{-3}$. It is well known that the energy per particle in the thermodynamic limit, $e_{0}(\rho)$, does not depend on the details of (reasonable) $\Omega$, so we are free to use a cube and take $N \rightarrow \infty$ through any sequence we please, as far as $e_{0}(\rho)$ is concerned. We set $N=k M$ with $k$ an integer and $M$ the cube of an integer, because we shall want to divide up $\Omega$ into $M$ smaller cubes (called cells) of length $\ell=(k / \rho)^{1 / 3}$. We will take $M \rightarrow \infty$ with $\ell$ and $k=\rho \ell^{3}$ fixed, but large.

The N-body Schrödinger operator is

$$
H=-\mu \sum_{i=1}^{N} \Delta_{i}+\sum_{1 \leq i<j \leq N} v\left(\mathbf{x}_{i}-\mathbf{x}_{j}\right) .
$$

For boundary conditions we impose Neumann (zero derivative) boundary conditions on $\Omega$. It is well-known that Neumann boundary conditions give the lowest possible g.s.e. for $H$, and hence its use is appropriate for a discussion of a lower bound for the g.s.e. Denote this Neumann g.s.e. by $E_{0}(N, L)$.

Now divide $\Omega$ into $M$ cells and impose Neumann conditions on each cell, which, as stated before, lowers the energy further. We also neglect the interaction between particles in different cells; this, too, can only lower the energy because $v \geq 0$.

A lower bound for $E_{0}(N, L)$ is obtained by distributing the $N$ particles in the $M$ cells and then finding a lower bound for the energy in these cells, which are now independent. We then add these $M$ energies. Finally, we minimize the total energy over all choices of the particle number in each cell (subject to the total number being $N)$. Despite the independence of the cells, the latter problem is not easy. In particular, something has to be invoked to make sure that we do not end up with some cells having too large a number of particles and some cells having too few.

With $L, N$ and $M=N /\left(\rho \ell^{3}\right)$ fixed, let $M c_{n}$, for $n=$ $0,1,2, \ldots$ denote the number of cells containing exactly $n$ particles. Then the particle number and cell number constraints are

$$
\sum_{n \geq 0} n c_{n}=k=\rho \ell^{3}, \quad \sum_{n \geq 0} c_{n}=1
$$

and our energy bound is

$$
E_{0}(N, L) \geq M \min \sum_{n \geq 0} c_{n} E_{0}(n, \ell),
$$

where the minimum is over all $c_{n} \geq 0$ satisfying (5).

The minimization would be easy if we knew that $E_{0}(n, \ell)$ (or a good lower bound for it) is convex in $n$, for then the optimum would be $c_{n}=\delta_{n, k}$. This convexity is very plausible, but we cannot prove it (except in the thermodynamic limit, where it amounts to thermodynamic stability). What we do know instead is superadditivity:

$$
E_{0}\left(n+n^{\prime}, \ell\right) \geq E_{0}(n, \ell)+E_{0}\left(n^{\prime}, \ell\right)
$$

for all $n, n^{\prime}$, and this turns out to be an adequate substitute for controlling the large $n$ terms in (6).

Eq. (7) is an immediate consequence of the positivity of the potential and it is used as follows. Suppose, provisionally, that we have a lower bound of the form 


$$
E_{0}(n, \ell) \geq K(\ell) n(n-1) \quad \text { for } 0 \leq n \leq 4 k,
$$

with $K(\ell)$ independent of $n$ for $0 \leq n \leq 4 k$. In fact, we shall later prove that for small enough $\rho$ (and hence small enough $k$ ) and suitable $\ell$, (8) holds with

$$
K(\ell) \geq \mu 4 \pi a \ell^{-3}\left(1-C^{\prime} Y^{1 / 17}\right),
$$

with $C^{\prime}$ some constant. [However, the analysis we give now, leading to (12), does not depend on this particular form of $K(\ell)$.]

Split the sum in (6) into two pieces: $0 \leq n<4 k$ and $4 k \leq n$. Let $t \equiv \sum_{n<4 k} n c_{n} \leq k$, so that $k-t=\sum_{n \geq 4 k} n c_{n}$. From (8) and Cauchy's inequality (and $\sum_{n<4 k} c_{n} \leq 1$ )

$$
\sum_{n<4 k} c_{n} E_{0}(n, \ell) \geq K(\ell) t(t-1) .
$$

On the other hand, if $n \geq 4 k$ then, by (7), $E_{0}(n, \ell) \geq$ $(n / 8 k) E_{0}(4 k, \ell)$, so

$$
\sum_{n \geq 4 k} c_{n} E_{0}(n, \ell) \geq \frac{k-t}{2} K(\ell)(4 k-1) .
$$

Upon adding (10) and (11) the factor $t(t-1)+(k-t)(2 k-$ $1 / 2)$ is obtained. Although the number $t$ is unknown, we note that this factor is monotone decreasing in $t$ in the interval $0 \leq t \leq k$ ( which is where $t$ lies, by (5)). Thus, we can set $t=k$ and obtain the same bound as if we had convexity, i.e.,

$$
E_{0}(N, L) \geq N K(\ell)\left(\rho \ell^{3}-1\right) .
$$

In summary, if we can show (8) for a box of a fixed size $\ell$, for all particle numbers up to $n=4 \rho \ell^{3}$, then we will have obtained our goal, (2), in the thermodynamic limit provided we can show that the $K$ in (8) satisfies (9) with the constant $C^{\prime}$ when $\ell$ is large compared to the mean particle spacing, i.e., $\rho \ell^{3}>C^{\prime \prime} Y^{-1 / 17}$. Then the $C$ in (2) is equals $C^{\prime}+C^{\prime \prime}$.

We now focus on a single cell and denote the $n$ coordinates $\left(\mathbf{x}_{1}, \ldots, \mathbf{x}_{n}\right)$ collectively by $\mathbf{X}$. The first step in proving (9) is to replace the total potential, $\sum_{i<j} v\left(\mathbf{x}_{i}-\mathbf{x}_{j}\right)$, by a lesser quantity, $\mathcal{W}_{v}(\mathbf{X})$, the nearest neighbor potential defined by

$$
\mathcal{W}_{v}(\mathbf{X}) \equiv \frac{1}{2} \sum_{i=1}^{n} v\left(\mathbf{x}_{i}-\mathbf{x}_{j(i)}\right),
$$

where $j(i)$ is the nearest neighbor to particle $i$ in the configuration $\mathbf{X}$. I.e., particle $i$ 'feels' only its nearest neighbor. Hence, we replace $H$ by the smaller operator

$$
\widetilde{H} \equiv \mathcal{T}+\mathcal{W}_{v} \leq H,
$$

where $\mathcal{T}=-\mu \sum \Delta_{i}$ is the kinetic energy in (伍). Since $v \geq 0$, the g.s.e. of $\widetilde{H}$ satisfies $\widetilde{E}_{0}(n, \ell) \leq E_{0}(n, \ell)$.
To get into the kinetic energy dominated region, we wish to replace $v$ in $(13)$ by a gentler potential $U$. To this end we generalize Lemma 1 of [1] and simplify its proof.

LEMMA 1: Let $v(r) \geq 0$ and $v(r)=0$ for $r>R_{0}$. Let $U(r) \geq 0$ be any function satisfying $\int U(r) r^{2} d r \leq 1$ and $U(r)=0$ for $r<R_{0}$. Let $\mathcal{B} \subset \mathbf{R}^{3}$ be star-shaped (convex suffices) with respect to 0 . Then, for all functions $\phi$,

$$
\int_{\mathcal{B}} \mu|\nabla \phi(\mathbf{x})|^{2}+\left[\frac{1}{2} v(r)-\mu a U(r)\right]|\phi(\mathbf{x})|^{2} d^{3} \mathbf{x} \geq 0 .
$$

Proof: Actually, (15) holds with $\mu|\nabla \phi(\mathbf{x})|^{2}$ replaced by the (smaller) radial kinetic energy, $\mu|\partial \phi(\mathbf{x}) / \partial r|^{2}$, and thus it suffices to prove the analog of (15) for the integral along each radial line, and to assume that $\phi(\mathbf{x})=u(r) / r$ along this line, with $u(0)=0$. Let us first prove (15) when $U$ is a delta-function at some radius $R \geq R_{0}$, i.e., $U(r)=R^{-2} \delta(r-R)$. Then, it is enough to show, for all $u$, that

$$
\int_{0}^{R} \mu\left|u^{\prime}(r)-u(r) / r\right|^{2}+\frac{1}{2} v(r)|u(r)|^{2} d r \geq \mu a|u(R)|^{2} R^{-2} .
$$

If the length of the radial line is less than $R$ then (16) is trivial. Otherwise, normalize $u$ by $u(R)=R-a$, and ask for the minimum of the left side of (16) under the condition that $u(0)=0, u(R)=R-a$. This is a simple problem in the calculus of variations and leads to the scattering length equation (3). If we substitute the solution into (16), integrate by parts, and note that $u_{0}(r)=r-a$ for $r>R_{0}$, we find that (16) is true if $a \leq R$, which is true since $u_{0} \geq 0$. Finally, by linearity and the fact that $U(r)=\int r^{-2} \delta(r-s) U(s) s^{2} d s$, the $\delta$-function case implies the general case. Q.E.D.

We select our $U$ by picking some $R \gg R_{0}$ and setting

$$
U(r)=3\left(R^{3}-R_{0}^{3}\right)^{-1} \quad \text { for } R_{0}<r<R
$$

and $U(r)=0$ otherwise. Later on we shall choose $R$, and we shall take

$$
R_{0} \ll R \ll \rho^{-1 / 3} \ll \ell .
$$

By further decomposing a cube into Voronoi cells (which are always convex), Dyson [1] deduces from Lemma 1 that $\widetilde{H}$ is bounded below by a nearest neighbor potential, as in (13), i.e.,

$$
H>\widetilde{H}>\mu a \mathcal{W}_{U}(\mathbf{X}),
$$

where $\mathcal{W}_{U}$ is as in (13), with $v$ replaced by $U$. For the hard core case, Dyson estimates the minimum (over all $\mathbf{X})$ of $\mathcal{W}_{U}(\mathbf{X})$, for a $U$ similar to (17), and gets a lower bound for all $\rho$, but 14 times smaller than (11). We follow another route. An important quantity for us will be the average value of $\mathcal{W}_{U}(\mathbf{X})$ in a cell, denoted by $\left\langle\mathcal{W}_{U}\right\rangle$. 
To compute $\left\langle U\left(\mathbf{x}_{1}-\mathbf{x}_{j(1)}\right)\right\rangle$, for example it is easiest to do the $\mathbf{x}_{2}, \ldots, \mathbf{x}_{n}$ integrations over the cell first and then the $\mathbf{x}_{1}$ integration. Provided $\mathbf{x}_{1}$ is in the smaller cube which is a distance $R$ from the cell boundary (whose volume is $\left.(\ell-2 R)^{3}\right)$, the probability that $R_{0}<\left|\mathbf{x}_{j}-\mathbf{x}_{1}\right|<R$ is $4 \pi\left(R^{3}-R_{0}^{3}\right) / 3 \ell^{3}$. Thus, performing the $\mathbf{x}_{1}$ integration over the smaller cube, and then adding similar contributions from $U\left(\mathbf{x}_{2}-\mathbf{x}_{j(2)}\right)$, etc., and using (17), we get

$$
\begin{aligned}
\left\langle\mathcal{W}_{U}\right\rangle & \geq \frac{3 n(\ell-2 R)^{3}}{\left(R^{3}-R_{0}^{3}\right) \ell^{3}}\left[1-(1-Q)^{n-1}\right] \\
& \geq \frac{4 \pi}{\ell^{3}} n(n-1)\left(1-\frac{2 R}{\ell}\right)^{3} \frac{1}{1+Q(n-1)} .
\end{aligned}
$$

with

$$
Q=4 \pi\left(R^{3}-R_{0}^{3}\right) / 3 \ell^{3} \ll 1 .
$$

In (21) we used $[1-x]^{n-1} \leq[1+(n-1) x]^{-1}$ for $0 \leq x \leq 1$. Note that (21) is of the form (8).

By similar reasoning, we obtain the upper bound

$$
\begin{aligned}
\left\langle\mathcal{W}_{U}\right\rangle & \leq \frac{3 n}{R^{3}-R_{0}^{3}}\left[1-(1-Q)^{n-1}\right] \\
& \leq \frac{4 \pi}{\ell^{3}} n(n-1) .
\end{aligned}
$$

Since $U(r)^{2}=4 \pi\left(Q \ell^{3}\right)^{-1} U(r)$, we also obtain

$$
\left\langle\mathcal{W}_{U}^{2}\right\rangle \leq 4 \pi n\left(Q \ell^{3}\right)^{-1}\left\langle\mathcal{W}_{U}\right\rangle
$$

We can now use Lemma 1 and these averages to obtain (8) and (9). Instead of using (19) alone, we pick some $0<\varepsilon \ll 1$ and, borrowing a bit of kinetic energy, define

$$
\widehat{H} \equiv \varepsilon \mathcal{T}+(1-\varepsilon) \mu a \mathcal{W}_{U}(\mathbf{X}) .
$$

By Lemma 1 and $v \geq 0$, we have

$$
H>\widetilde{H}>\widehat{H}
$$

We shall derive (8) and (9) from a lower bound to $\widehat{H}$.

Although $\varepsilon$ is small, we regard $H_{0} \equiv \varepsilon \mathcal{T}$ as our unperturbed Hamiltonian and $V \equiv(1-\varepsilon) \mu a \mathcal{W}_{U}(\mathbf{X})$ as a perturbation of $H_{0}$. The ground state wave function for $H_{0}$ is $\Psi_{0}(\mathbf{X})=\ell^{-3 n / 2}$ and $H_{0} \Psi_{0}=\lambda_{0} \Psi_{0}=0$ (Neumann conditions). The second eigenvalue of $H_{0}$ is $\lambda_{1}=\varepsilon \mu \pi / \ell^{2}$. Note that the ground state expectation, $\left\langle\Psi_{0}\left|\mathcal{W}_{U}\right| \Psi_{0}\right\rangle$, is precisely the average $\left\langle\mathcal{W}_{U}\right\rangle$ mentioned in (20)-(25).

Temple's inequality [13] states that when a perturbation $V$ is non-negative (as here) and when $\lambda_{1}-\lambda_{0} \geq$ $\left\langle\Psi_{0}|V| \Psi_{0}\right\rangle$ then the g.s.e., $E_{0}$, of the perturbed Hamiltonian $H=H_{0}+V$ satisfies

$$
E_{0} \geq \lambda_{0}+\left\langle\Psi_{0}|V| \Psi_{0}\right\rangle-\frac{\left\langle\Psi_{0}\left|V^{2}\right| \Psi_{0}\right\rangle-\left\langle\Psi_{0}|V| \Psi_{0}\right\rangle^{2}}{\lambda_{1}-\lambda_{0}-\left\langle\Psi_{0}|V| \Psi_{0}\right\rangle} .
$$

We apply this to our case with $\lambda_{1}-\lambda_{0}=\varepsilon \mu \pi / \ell^{2}$ and $V=(1-\varepsilon) \mu a \mathcal{W}_{U}$. We neglect the (positive) term
$\left\langle\Psi_{0}|V| \Psi_{0}\right\rangle^{2}$ in (28) and we use (21), (24) and (25). We also use $1-\varepsilon<1$ in two appropriate places and find

$$
\frac{E_{0}(n, \ell)}{\mu a\left\langle\mathcal{W}_{U}\right\rangle} \geq(1-\varepsilon)\left(1-\frac{4 \pi a n}{Q \ell} \frac{1}{\varepsilon \pi-a \ell^{2}\left\langle\mathcal{W}_{U}\right\rangle}\right) \text {. }
$$

Apart from some higher order errors, $(29)$ is just what we need in (8), (9). Let us denote the order of the main error by $Y^{\alpha}$, and we would like to show that $\alpha=1 / 17$ suffices. The errors are the following:

From the $(1-\varepsilon)$ factor, we need $\varepsilon \leq O\left(Y^{\alpha}\right)$.

From the $Q(n+1)$ error in (21) we need $Q \rho \ell^{3} \leq O\left(Y^{\alpha}\right)$.

From the $R / \ell$ error in (21) we need $R / \ell \leq O\left(Y^{\alpha}\right)$.

From (29) we need $a \ell^{2}\left\langle\mathcal{W}_{U}\right\rangle / \varepsilon \leq O\left(Y^{\alpha}\right)$ and $\rho \ell^{5} a /\left(R^{3} \varepsilon\right) \leq O\left(Y^{\alpha}\right)$.

All these desiderata can be met with $\varepsilon=Y^{\alpha}, R / \ell=$ $Y^{\alpha}, Q=O\left(Y^{\alpha}\right), \rho R^{3}=Y^{2 \alpha}$ and $\alpha=1 / 17$ - as claimed.

The partial support of U.S. National Science Foundation grant PHY95-13072A01 (EHL) and the Adalsteinn Kristjansson Foundation of the University of Iceland (JY) is gratefully acknowledged.

[1] F.J. Dyson, Phys. Rev. 106, 20 (1957).

[2] W. Ketterle and N. J. van Druten, in B. Bederson and H. Walther, eds., Advances in Atomic, Molecular and Optical Physics, 37, 181, Academic Press (1996).

[3] B. Baumgartner, J. Phys. A 30, L741 (1997).

[4] J. Oliva, Phys. Rev. B 39, 4197 (1989).

[5] F.J. Dyson, J. Math. Phys. 8, 1538 (1967).

[6] J.G. Conlon, E.H. Lieb and H-T. Yau, Commun. Math. Phys. 116, 417 (1988).

[7] N.N. Bogoliubov, J. Phys. (U.S.S.R.) 11, 23 (1947); N.N. Bogoliubov and D.N. Zubarev, Sov. Phys.-JETP 1, 83 (1955).

[8] K. Huang and C.N. Yang, Phys. Rev. 105, 767-775 (1957); T.D. Lee, K. Huang and C.N. Yang, Phys. Rev. 106, 1135-1145 (1957); K.A. Brueckner and K. Sawada, Phys. Rev. 106, 1117-1127, 1128-1135 (1957).; S.T. Beliaev, Sov. Phys.-JETP 7, 299-307 (1958); T.T. Wu, Phys. Rev. 115, 1390 (1959); N. Hugenholtz and D. Pines, Phys. Rev. 116, 489 (1959); M. Girardeau and R. Arnowitt, Phys. Rev. 113, 755 (1959); T.D. Lee and C.N. Yang, Phys. Rev. 117, 12 (1960).

[9] E.H. Lieb, Phys. Rev. 130, 2518 (1963); E.H. Lieb and A.Y. Sakakura, Phys. Rev. 133, A899 (1964); E.H. Lieb and W. Liniger, Phys. Rev. 134, A312 (1964).

[10] E.H. Lieb, Proc. Nat. Acad. Sci. (U.S.) 46, 1000 (1960).

[11] E.H. Lieb, The Bose fluid, in Lecture Notes in Theoretical Physics VIIC, W.E. Brittin, ed., Univ. of Colorado Press, pp. 175 (1964).

[12] E.H. Lieb, T. Kennedy and S. Shastry, Phys. Rev. Lett. 61, 2582-2584 (1988).

[13] G. Temple, Proc. Roy. Soc. London A 119, 276 (1928). 